\title{
Identification and Optimal Estimation of Random Fields from Scattered Point-wise Data*
}

\author{
G. BASTIN† and M. GEVERS †t
}

\begin{abstract}
Procedures are available for the estimation of various functionals of a random field in the practically meaningful case where the random field is possibly non-stationary and where the data are scarce and randomly scattered in space.
\end{abstract}

Key Words-Random fields; estimation; interpolation; water resources.

\begin{abstract}
A self-contained presentation of the interpolation problem in two-dimensional spatial random fields is given. We investigate the case where the random field is not necessarily stationary, where the data are so scarce and so scattered in space that sample covariance function estimates are not meaningful and where, therefore, an analytical parametric 'variogram' model is used in lieu of the covariance. We discuss several variogram parameter estimation techniques (LS, GLS, ML, Interpolation Error Method) and we show how to derive estimates of various functionals of the random field from the variogram. A numerical simulation and two typical engineering applications illustrate the variogram estimation techniques and provide a good measure of their respective performances.
\end{abstract}

\section{INTRODUCTION}

IN THIS paper we discuss the problem of interpolation in two-dimensional (2-D) random fields (RF) with scattered data. Our interpolators are linear minimum variance unbiased estimators (often called BLUE, for Best Linear Unbiased Estimator), and the reader might justifiably argue that the theory of linear minimum variance estimation in random fields is well established. The point, however, is that we are concerned with reallife problems, where the mean and the covariance function of the RF are not known a priori, and where the available data are so scattered (and often so scarce) that sample estimates of these functions would be completely meaningless. The major difficulty, then, is the preliminary identification of a mathematical model of the RF. The major thrust of our contribution is therefore concerned with this identification problem.

* Received 9 March 1983; revised 4 January 1984; revised 23 April 1984. The original version of this paper was not presented at any IFAC meeting. This paper was recommended for publication in revised form by associate editor T. Başar under the direction of editor H. Kwakernaak.

† Laboratoire d'Automatique et d'Analyse des Systemes, Louvain University, Bâtiment Maxwell, B-1348 Louvain-laNeuve, Belgium.

† Present address: Department of Systems Engineering Institute of Advanced Studies, Australian National University, Canberra, Australia.
We consider a 2 -D RF $Z(x, y)$ over a domain $\Omega:(x, y) \varepsilon \Omega \subseteq R^{2}$, and we assume that a realization of this RF is available in the form of a finite-dimensional vector of measurements $\mathbf{Z}=\left(z_{1}, \ldots, z_{N}\right)$, where $z_{i}=z\left(x_{i}, y_{i}\right)$ is a realization of $Z\left(x_{i}, y_{i}\right)$. The $N$ locations are randomly scattered in the domain $\Omega$ (Fig. 1). We want to construct an optimal linear estimator $\hat{f}(z)$ for various functionals $f(z)$ of the RF

$$
\hat{f}(z)=\lambda_{0}+\sum_{i=1}^{N} \lambda_{i} z_{i}
$$

Before we consider this estimation problem, we shall give a few examples for $f(z)$. This will at the same time illustrate the kind of applications that this paper addresses itself to.

(i) $f(z)=z\left(x_{0}, y_{0}\right)$ with $\left(x_{0}, y_{0}\right) \in \Omega$, i.e. we want to estimate the value of the RF at a fixed but arbitrary point of the domain. A typical application is in contour mapping (or cartography): for example, one may want to estimate the rainfall $Z\left(x_{i}, y_{i}\right)$ at various points of a region (or at all points of a grid covering a region) from a small number of observations in rain-gauges scattered over that region (Delfiner and Delhomme, 1975). An application of contour mapping will be presented in Section 6.

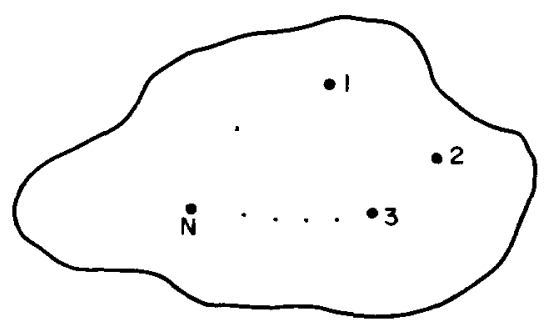

FIG. 1. Data locations randomly scattered in $\Omega$. 
(ii) $f(z)=\frac{1}{S_{\Omega}} \int_{\Omega} z(x, y) \mathrm{d} x \mathrm{~d} y$, where $S_{\Omega}$ is the surface area of the domain $\Omega$. With the same application in mind as before, one may want to estimate the average rainfall over an area from $N$ available measurements (Lorent et al., 1982): see Section 7. Another typical example is in geophysics where one wants to estimate the mean ore grade of a mineral field from a few available measurements (David, 1977).

$$
\text { (iii) } f(z)=\left|\frac{\partial z}{\partial x}, \frac{\partial z}{\partial y}\right|_{\left(x_{0}, y_{0}\right)}^{T} \text {, the derivative of the RF }
$$

at a point $\left(x_{0}, y_{0}\right)$. A typical application is in groundwater flow modelling, where the evaluation of the gradient of the water level is necessary at all nodes of a grid in order to identify the flow equation (Bastin and Duque, 1981). It is then required to estimate the gradient from a limited number of water level measurements, which are most often not located at the grid nodes.

Some common features in many such applications are the following:

- Measurements are available in only a very limited number of locations, which are not equispaced. This contrasts with 2-D estimation problems in image processing where the data points are numerous and located on a grid.

-The mean of the RF is almost never zero.

- The mean and the spatial covariance function are seldom known and are hard to estimate, precisely because the measure points are few and not evenly spaced.

- - The RF is often not wide-sense stationary, or at least a wide-sense stationary assumption is hard to validate from the data.

These features contrast with time-series processes, much better known to control engineers, which can most often be assumed stationary and ergodic, and in which the measurements are often numerous and almost always equispaced (in time). In addition, the mean can often be assumed zero. When the mean is unknown, it must be estimated, thereby introducing a bias in the covariance estimate. However, both the mean and the covariance function can usually be estimated fairly precisely because of the large number of available measurements, the fixed sampling interval and the ergodicity property.

The presence of a non-zero mean and the nonstationarity of the RF has led the geostatisticians to suggest the use of the 'variogram function' as an alternative to the covariance function for interpolation in random fields. The scarcity of the data requires the use of analytic variogram models, and the development of techniques for the estimation of these models. This, together with the applications of Sections 6 and 7, is the main contribution of this paper.
The applications that we have mentioned so far are typical systems engineering applications. Such applications have been numerous in geostatistics for quite some time: the books by David (1977) and Journel and Huijbrechts (1978) contain many references. More recently, the theory has been further developed and applied to problems in hydrology (Delfiner and Delhomme, 1975; De Wrachien, 1976; Delhomme, 1978; Volpi and Gambolati, 1978; Gambolati and Volpi, 1979: Darricau, 1979; Gevers and Bastin, 1980; Darricau, 1980; Chirlin and Dagan, 1980; Chua and Bras, 1982; Creutin and Obled, 1982; Lorent et al., 1982, Kitanidis, 1983).

Yet, despite their wide range of possible applications, the methods that we shall present are almost unknown to systems engineers. The foundations of the theory of 2-D interpolation on which this paper is based have been established by a South African mining engineer Krige (1951), and in geostatistics and hydrosciences this 2-D interpolation technique is often referred to as kriging. The theory has been further developed to a great extent by the French geostatistician Matheron $(1965,1973)$. Our own interest in this theory originated with a parameter estimation problem in 2-D groundwaterflow models. In fact, when the parameters of a distributed parameter system must be estimated, it is often necessary to apply a 2-D interpolation method as a first step, because the measurements are not available at all points of the domain (see e.g. Gevers and Bastin, 1982). Over the years, we have refined some theoretical points, studied different approaches for the identification of the RF model and applied this technique to a large number of problems, particularly in the field of hydrology. A software package for 2-D minimum variance unbiased interpolation and automatic cartography has also been developed.

The purpose of this paper is to give a selfcontained and hopefully informative presentation of this 2-D interpolation technique and to report on some real-life applications that we have dealt with.

The paper is organized as follows. In Section 2 we give the expressions of the BLUE for the case of point wise interpolation (i.e. $f(z)=z\left(x_{0}, b_{0}\right)$ ). The interpolation formulas for other functionals $f(z)$ are easy to derive, and an application in Section 7 will illustrate the case where $f(z)$ is the spatial average of the RF. Two different methods for the estimation of $f(z)$ are presented: one where the mean and the autocovariance function of the RF are assumed stationary and known, the other where the mean is unknown but the variogram is stationary and known. In Section 3 we show why the second method should be preferred, and we illustrate our argument with a typical application. Now. writing a BLUE as a function of the variogram is an easy task : 
the most difficult part of a 2-D estimation problem is the identification of a model for the variogram. This is the object of Section 4 where we present different methods for the estimation of a variogram model. In Section 5 , a very simple numerical simulation is used to show how meaningless the sample (or experimental) variogram can be; it also illustrates the superiority of the interpolation error method of Section 4 over the classical least-squares technique for the estimation of the variogram model parameters. In Section 6, we apply the 2-D interpolation technique to the contour mapping of the water level in a groundwater reservoir. This application illustrates that the use of the experimental variogram (or covariance) for 2-D interpolation leads to absurd values for the water level estimates. Four different analytic variogram models and two parameter estimation techniques are compared in this application. In Section 7, we present an application where the estimated quantity is the surface integral of the measured variable, namely the average rainfall over an area. This is an interesting illustration of the theory, because the RF is now also a function of time, the average areal rainfall being an ordinary time series. We shall see that the variogram can be factored into the product of a time-dependent scaling factor, and a geometrydependent shaping factor. This has enabled us to propose a procedure for the optimal selection of rain-gauge locations. The applications presented here have been kept short, because they mainly serve to illustrate the theory. Full details about these applications have been published elsewhere: see the reference list.

The use of the variogram function for 2-D interpolation in RF has been common practice in geostatistics and hydrology: see the references already mentioned, and in particular the pioneering work of Matheron. To our knowledge, in the applications published so far, the identification of the analytical variogram model has almost always been performed by intuitive fitting or by leastsquares fitting of an analytic variogram model to the so-called 'experimental variogram' (see Sections 3 and 4). Our own contribution has been in the development of alternative estimation techniques for variogram parameters (the Generalized LeastSquares, Interpolation Error and Maximum Likelihood methods presented in Section 4) and in the applications of Sections 6 and 7 . The reason for looking at alternative estimation techniques was the obvious lack of robustness of the widely used leastsquares technique in the case of scarce data. This is illustrated by the theoretical example of Section 4.4, the simulation of Section 5 and the application of Section 6. The identification criterion of the Interpolation Error Method (Section 4.6) is closely related to the criterion of the 'a posteriori validation' approach used at the Ecole des Mines de Paris (see e.g. Delhomme, 1976).

\section{OPTIMAL INTERPOLATION IN A RANDOM FIELD}

Assumptions, notations and definitions

We consider a real-valued $\mathrm{RF} Z(x, y)$, $(x, y) \in \Omega \subseteq R^{2}$, whose deviations from the mean are purely nondeterministic, and for which the following functions are defined:

- The mean (assumed constant)

$$
m=E\{z(x, y)\}
$$

- The spatial covariance kernel

$$
R(i, j)=E\left\{\left[z\left(x_{i}, y_{i}\right)-m\right]\left[z\left(x_{j}, y_{j}\right)-m\right]\right\}
$$

where $\left(x_{i}, \dot{y}_{i}\right)$ and $\left(x_{j}, y_{j}\right)$ are two arbitrary points in $\Omega$.

-The spatial variogram (which is the name given by geostatisticians, see e.g. Journel and Huijbregts, 1978 , to the semi-variance of RF increments)

$$
\gamma(i, j)=\frac{1}{2} E\left\{\left[z\left(x_{i}, y_{i}\right)-z\left(x_{j}, y_{j}\right)\right]^{2}\right\}
$$

We consider two special classes of random fields:

(a) (Weak sense) stationary random fields. In addition to the stationary mean assumption, we assume that the covariance is isotropic and stationary and that its power spectrum $F(\lambda)$ is absolutely continuous. We denote

$$
R(i, j)=R\left(d_{i j}\right)
$$

where $d_{i j}$ is the Euclidean distance between the points $\left(x_{i}, y_{i}\right)$ and $\left(x_{j}, y_{j}\right)$. In this case, the RF variance is finite and stationary

$$
\sigma^{2}=R(0)
$$

and the variogram is, by definition, also stationary and related to the covariance function as follows:

$$
\gamma(d)=\sigma^{2}-R(d) \text {, with } \gamma(i, j)=\gamma\left(d_{i j}\right)
$$

By the absolute continuity assumption on the spectrum, we have (see e.g. Cramer and Leadbetter, 1967)

$$
\lim _{d \rightarrow \infty} R(d)=0 \text { and } \lim _{d \rightarrow \infty} \gamma(d)=\sigma^{2}=R(0) .
$$

(b) Intrinsic random fields. In addition to the stationary mean assumption, we assume that the 
variogram (but not necessarily the covariance) is isotropic and stationary:

$$
\gamma(i, j)=\gamma\left(d_{i j}\right)
$$

This is a wider class than class (a), since it involves not only stationary RF but also nonstationary RF with stationary increments (like e.g. 2-D Wiener fields). In this last case, the relationship (7) is no longer valid; the RF variance can be infinite, and the variogram can become unbounded:

$$
\lim _{d \rightarrow \infty} \gamma(d)=\infty
$$

\section{Optimal interpolation}

We consider now the following situation (Fig. 1). Given a finite realization $\mathbf{Z}=\left(z_{1}, \ldots, z_{N}\right)$ of the $\mathrm{RF}$ measured at $N$ scattered points in $\Omega$, find an optimal linear minimum variance unbiased estimate of $z\left(x_{0}, y_{0}\right)$ at an arbitrary point $\left(x_{0}, y_{0}\right) \in \Omega$. We solve this problem both for stationary and intrinsic random fields.

Method 1. Stationary fields with known mean and covariance. The linear minimum variance estimator for $z\left(x_{0}, y_{0}\right)$ is given by (see e.g. Papoulis, 1965)

$$
\hat{z}_{0} \equiv \hat{z}\left(x_{0}, y_{0}\right)=v_{0}+\sum_{i=1}^{N} v_{i} z_{i}=m+\sum_{i=1}^{N} v_{i}\left(z_{i}-m\right)
$$

where $\left\{v_{i}, i=1, \ldots, N\right\}$ is the solution of the system:

$$
\sum_{j=1}^{N} v_{j} R\left(d_{i j}\right)=R\left(d_{0 i}\right) \quad i=1, \ldots, N
$$

The interpolation error variance is given by

$$
\sigma_{0}^{2}=\sigma^{2}-\sum_{i=1}^{N} v_{i} R\left(d_{0 i}\right)
$$

This estimator is unbiased and is a straightforward extension of the well known Levinson predictor for stochastic processes to the case of interpolation in 2D RF (see e.g. Anderson and Moore, 1979). Note that (10) and (11) are also valid for nonstationary RF with known mean and covariance but it is of little practical use: situations where a nonstationary covariance is known are rather rare.

Method 2. Intrinsic fields with unknown mean and known variogram. We look for a linear minimum variance unbiased estimator of the form:

$$
\hat{z}_{0}=\lambda_{0}+\sum_{i=1}^{N} \lambda_{i} z_{i}
$$

with $\lambda_{i}$ idependent of the unknown mean $m$. The unbiasedness condition imposes

$$
\sum_{i=1}^{N} \lambda_{i}=1 \text { and } \lambda_{0}=0
$$

Minimization of $E\left\{\left(z_{0}-\hat{z}_{0}\right)^{2}\right\}$, with respect to $\left\{\lambda_{i}, i=1, \ldots, N\right\}$ under the constraints (14) leads to the system

$$
\begin{gathered}
\sum_{j=1}^{N} \lambda_{j} \gamma\left(d_{i j}\right)+\mu= \\
\gamma\left(d_{0 i}\right) \quad i=1, \ldots, N \\
\sum_{j=1}^{N} \lambda_{j}=1
\end{gathered}
$$

where $\mu$ is a Lagrange parameter.

The interpolation error variance is given by

$$
s_{0}^{2}=\mu+\sum_{i=1}^{N} \lambda_{i} \gamma\left(d_{0 i}\right)
$$

Remark: Evidently, method 2 can also be viewed as a suboptimal interpolation method in the case of stationary fields with known mean and covariance. In such a case, the following relationship between $\sigma_{0}^{2}$ and $s_{0}^{2}$ can be established (Bastin and Henriet, 1982; Gevers 1984)

$$
s_{0}^{2}=\sigma_{0}^{2}+\frac{\left(1-\mathbf{U}^{\mathrm{T}} R^{-1} \mathbf{R}_{0}\right)^{2}}{\mathbf{U}^{\mathrm{T}} R^{-1} \mathbf{U}}
$$

where

$$
\begin{aligned}
& R \text { is a } N \times N \text { matrix with entries } R\left(d_{i j}\right) \\
& \quad(i, j=1, \ldots, N) \\
& \mathbf{R}_{0} \text { is a column } N \text {-vector with entries } R\left(d_{0 i}\right) \\
& \quad(i=1, \ldots, N) \\
& U \text { is a column } N \text {-vector with entries } 1 .
\end{aligned}
$$

Clearly, in such case, $s_{0}^{2} \geqslant \sigma_{0}^{2}$.

\section{WHY SHOULD WE USE METHOD 2?}

In Section 2, we have presented two methods for the optimal interpolation of random fields. The basic assumptions are as follows:

For method 1 : (Weak sense) stationary assumption; the covariance of the RF $R(d)$ is stationary; the mean $m$ and the covariance $R(d)$ are known.

For method 2: Intrinsic assumption;

the variogram of the RF $\gamma(d)$ is stationary; the variogram $\gamma(d)$ is known.

In this section we shall give a number of arguments to show that it is actually preferable to use method 2 on real life interpolation problems.

In most practical applications, indeed, one does not know a priori if the RF is stationary or not, and 
the values of $m, R(d)$ or $\gamma(d)$ are not given. The only available information on the RF is the set of data. From the set of data, it is then necessary to:

(a) make a stationarity assumption;

(b) estimate the mean $m$ and the covariance $R(d)$ or the variogram $\gamma(d)$.

The first step in the analysis can be to draw the 'experimental variogram' of the RF.

\section{Computation of the experimental variogram}

The experimental variogram is obtained by computing the mean square differences between measured values of $z$ taken at all couples of points that are separated by a same distance. For a distance $d$

$$
\hat{\gamma(d)}=\frac{1}{2 N(d)} \sum_{k, j}\left(z_{k}-z_{j}\right)^{2}
$$

where the sum is taken over all couples of points $(k, j)$ separated by a distance $d$, and where $N(d)$ represents the number of such couples.

Except perhaps in mining applications where the drillings are often regularly spaced, $N(d)$ will usually be 0 or 1 . Therefore, in order to obtain a more representative picture, the following device is used. The interval of useful distances is divided in $m$ subintervals $\left[d_{i}, d_{i+1}\right], i=1, \ldots, m$, and for each subinterval the following estimator is used

$$
\hat{\gamma}\left(d_{i}\right)=\frac{1}{2 N_{i}} \sum_{k, j}\left(z_{k}-z_{j}\right)^{2}
$$

where the sum is over all couples of points $(k, j)$ separated by a distance $d$ such that $d_{i} \leqslant d<d_{i+1}, N_{i}$ is the number of such couples, and $\bar{d}_{i}=1 / 2$ $\left(d_{i}+d_{i+1}\right)$.

The experimental variogram has the graphical appearance of a broken line: a typical example is shown on Fig. 2, where the experimental variogram of a piezometric field is drawn. (See Section 6 for details.)

\section{Comparison between methods 1 and 2}

First notice that method 2 can be used in all cases where method 1 can be used but the converse is not true. Therefore the questions we face are:

-Are we allowed to use method 1 ? i.e. can we check whether the RF is stationary?

-If the RF is wide-sense stationary, does method 1 , in practice, have any advantage over method 2 ? The following comments can be made.

(1) In 2-D applications with scarce data, a stationarity assumption on the covariance is difficult to validate. With the standing assumptions (see Section 2), a stationary covariance requires

$$
\lim _{d \rightarrow \infty} \gamma(d)=\text { constant }=\sigma^{2} .
$$

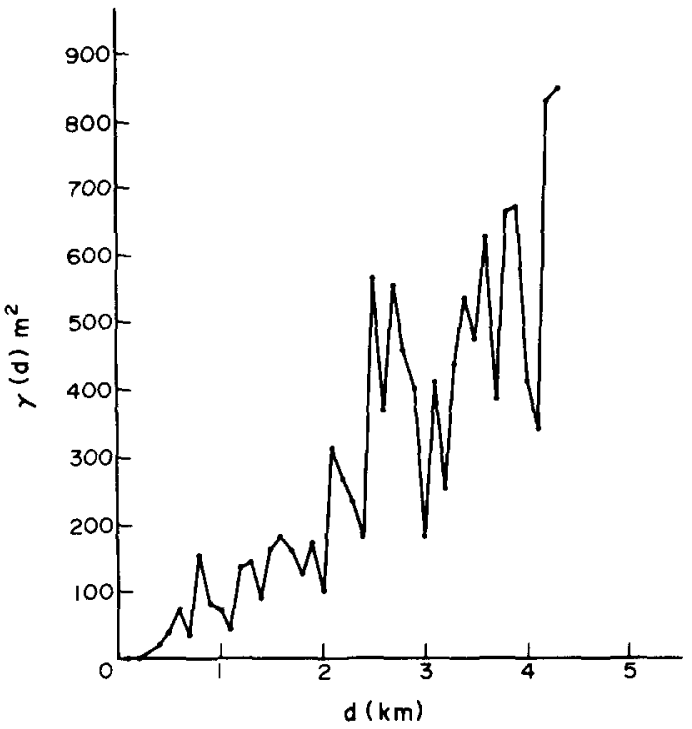

FIG. 2. Experimental variogram of the water level (piezometry) in a $6 \times 6 \mathrm{~km}$ aquifer.

In practice, the range of available distances is often too small to decide whether the variogram would reach a constant value for large $d$ : Fig. 2 illustrates this. Worse, in case of a nonstationary RF with stationary increments, the procedures of statistical inference which are used for the estimation of the sample covariance will make the hypothesis of a stationary covariance plausible, even though the RF has an infinite variance. See e.g. Matheron (1971) for an illuminating example.

(2) It is just as difficult to validate a stationarity assumption on the variogram. However, since a stationary variogram exists for a wider class of RF than a stationary covariance, it is always safer to opt for method 2 in case of doubt (see, e.g., the experimental variogram of Fig. 2). Notice in particular that the variogram can be stationary without any condition on $\lim _{d \rightarrow \infty} \gamma(d)$.

(3) Suppose nevertheless that the RF were known to be wide-sense stationary. Then

-if $m$ and $R(d)$ are known, method 1 is better than method 2: see (17).

-if $R(d)$ is known, but $m$ is unknown and is replaced in (10) by its sample mean, then method 2 yields a smaller interpolation error variance than method 1 : see Gevers (1984).

In the more realistic situation where $m$ and $R(d)$ are both unknown, the following can be shown (see Gevers (1984) for a time-series version of this result). Suppose a parametric variogram model $\hat{\gamma}(d)$ is estimated using one of the methods of Section 4 , and a corresponding analytic covariance model is derived from it, using

$$
\hat{R}(d)=\left[\lim _{d \rightarrow \infty} \hat{\gamma}(d)\right]-\hat{\gamma}(d) .
$$


Note: In the stationary case, the variogram model would be chosen such that $\lim _{d \rightarrow \infty} \gamma(d)$ is bounded.

Suppose further that a minimum variance unbiased estimate $\hat{m}$ is computed from the data using the above $\hat{R}(d)$ as the covariance function. Then the estimator $(10)$ of $\hat{z}_{0}$, with $m$ and $R(d)$ replaced by $\hat{m}$ and $\hat{R}(d)$, coincides with the estimator (13) of $\hat{z}_{0}$, with $\gamma(d)$ replaced by $\hat{\gamma}(d)$, i.e. with this estimation procedure for the statistics of the RF, the two methods are equivalent in the stationary case. Since method 2 is computationally simpler, and since it also applies to some nonstationary random fields, it is to be preferred in practice.

\section{ESTIMATION OF THE VARIOGRAM MODEL}

\subsection{Introduction}

In most applications, the number of equispaced measure points is so small that it is difficult to obtain a reasonable estimate of the variogram from the available data. Using the 'experimental variogram' (see Fig. 2) in the interpolation formulas (15) may lead to an ill-conditioned coefficient matrix and, in most cases, to completely absurd values for the interpolates $\hat{z}$; the application in Section 6 will illustrate this point. It is therefore necessary to use analytic variogram models, and to infer the parameters of these models from the data.

In this section, we shall briefly define the class of admissible variogram models, present some commonly used models and describe various estimation methods for the parameters of the variogram models. These methods can be subdivided into two classes:

(i) either the parameters are adjusted so that the variogram model fits the experimental variogram in some sense; or

(ii) or they are adjusted so as to minimize, in some sense, the interpolation errors computed with this variogram model.

\subsection{The class of admissible variogram models}

It is well known that a real function $R(d)$ is an admissible autocovariance function for a wide-sense stationary RF if and only if it is nonnegative definite, i.e.

$$
\sum_{i, j} \zeta_{i} \zeta_{j} R\left(d_{i j}\right) \geqslant 0
$$

for any set of arbitrary points $\left(x_{i}, y_{i}\right)$ and arbitrary numbers $\zeta_{i}$. In fact, within the framework of BLU interpolation, the function $R(d)$ must be strictly positive definite for the system (11) to be nonsingular

$$
\sum_{i, j} \zeta_{i \zeta j} R\left(d_{i j}\right)>0
$$

Furthermore, if $R(d)$ is a positive definite covariance function, then the following inequalities are satisfied (Papoulis, 1965)

(a) $|R(d)|<R(0)$ for $d>0$

(b) $4^{n}[R(0)-R(d)]<R(0)-R\left(4^{n} d\right)$ for $d>0$.

Clearly, these inequalities are necessary (but not sufficient) conditions for $R(d)$ to be a covariance.

For intrinsic RF (with possibly infinite variance), a wider class of functions are admissible as variograms. Following similar arguments as for the covariance, it can be shown (e.g. Matheron, 1965) that a real function $\gamma(d)$ is an admissible variogram if and only if

$$
-\sum_{i, j} \zeta_{i} \zeta_{j} \gamma\left(d_{i j}\right)>0 \quad \text { with } \sum_{i} \zeta_{i}=0
$$

for any set of arbitrary data points and arbitrary zero-sum numbers $\zeta_{i}$. If a function $\gamma(d)$ has this property, then $-\gamma(d)$ has been called 'conditionally positive definite' (Matheron, 1971). Note that the interpolation error variance $s_{0}^{2}$ can be written

$$
s_{0}^{2}=-\left(\lambda^{t}-1\right)\left(\begin{array}{ll}
\Gamma & \Gamma_{0} \\
\Gamma_{0}^{\mathrm{T}} & 0
\end{array}\right)\left(\begin{array}{r}
\lambda \\
-1
\end{array}\right) .
$$

Therefore, if $-\gamma(d)$ is conditionally positive definite. then $s_{0}^{2}$ is guaranteed to be positive for all possible locations of data points. As for the covariance, it is easy to derive the following necessary conditions for an admissible variogram

$$
\begin{aligned}
& \gamma(0)=0 \\
& \gamma(d)>0 \text { for } d>0 \\
& \gamma\left(2^{n} d\right)<4^{n} \gamma(d) \text { for } d>0 .
\end{aligned}
$$

\subsection{Commonly used variogram models}

The range of admissible parametric variogram models is of course endless, but the shape of the experimental variogram obtained in numerous practical applications indicates that fairly simple models can be used. The most commonly used variogram models are (Matheron, 1965; Delhomme, 1976):

$$
\begin{aligned}
\gamma(d) & =\alpha \mathrm{d}^{\beta} \\
\gamma(d) & =\alpha[1-\exp (-\beta d) \\
\gamma(d) & =\alpha\left[1-\exp \left(-\beta d^{2}\right)\right] \\
\gamma(d) & =\alpha \log (1+\beta d) .
\end{aligned}
$$

The necessary conditions (25) imply that $\alpha>0$ and $\beta>0$ for these four models, and furthermore that $\beta<2$ for the model (26a). Note that all these models have the general form

$$
\gamma(d ; \alpha, \beta)=\alpha \gamma^{*}(d ; \beta) .
$$


With this form, $\alpha$ is called the 'scale factor' while $\gamma^{*}(d ; \beta)$ is called the shaping factor or the spatial autocorrelation factor. Then it is important to observe that:

(a) the optimal interpolator $\hat{z}_{0}=\sum_{i=1}^{N} \lambda_{i} z_{i}$ is independent of the scale factor, i.e. the $\lambda_{i}$ coefficients are independent of $\alpha$, but depend only on the shaping factor (see (15a));

(b) the variance of the interpolation error (16) can be written as

$$
s_{0}^{2}=\alpha V^{*}(\beta) ; \text { and }
$$

(c) in the case of a stationary field, with finite variance $\sigma^{2}$ and covariance $R(d)$, one can identify

$$
\begin{gathered}
\alpha=\sigma^{2} \\
\gamma^{*}(d ; \beta)=1-\mathbf{R}(\mathrm{d}) / \sigma^{2} .
\end{gathered}
$$

This clearly justifies the name of 'autocorrelation factor' for $\gamma^{*}(d ; \beta)$.

\subsection{Estimation of $\alpha$ and $\beta$ by the least squares method}

Given the measurements $z_{1}, \ldots, z_{N}$, one can compute experimental squared increments

$$
q_{i j}=\frac{1}{2}\left(z_{i}-z_{j}\right)^{2} \quad i=1, \ldots N ; j=i+1, \ldots, N
$$

$q_{i j}$ is an unbiased estimate of $\gamma(i, j)$ and we can write

$$
q_{i j}=\gamma\left(d_{i j}\right)+v_{i j} \text { with } \mathrm{E}\left[v_{i j}\right]=0 .
$$

Having chosen a theoretical model $\gamma(d ; \alpha, \beta)$, the parameters $\alpha$ and $\beta$ are then obtained by minimizing the cost function

$$
J(\alpha, \beta)=\sum_{i=1}^{N} \sum_{j=i+1}^{N}\left\{q_{i j}-\alpha \gamma^{*}\left(d_{i j} ; \beta\right)\right\}^{2}
$$

Since $J(\alpha, \beta)$ is quadratic in $\alpha, \partial J / \partial \alpha=0$ yields

$$
\hat{\alpha}_{\mathrm{LS}}(\beta)=\frac{\Sigma \Sigma q_{i j} \gamma^{*}\left(d_{i j}, \beta\right)}{\sum \Sigma\left[\gamma^{*}\left(d_{i j}, \beta\right)\right]^{2}}
$$

$\beta$ is then obtained by minimizing

$$
\begin{aligned}
J^{*}(\beta)= & J\left(\hat{\alpha}_{L S}(\beta), \beta\right), \\
& =\sum_{i} \sum_{j}\left\{q_{i j}-\hat{\alpha}_{L S}(\beta) \gamma^{*}\left(d_{i j}, \beta\right)\right\}^{2} .
\end{aligned}
$$

We conclude that the estimation of $\alpha$ and $\beta$ can be decoupled: $\hat{\beta}$ is estimated first by minimizing (34), then $\hat{\alpha}$ is computed by (33).
Although very simple in principle, the leastsquares method has a serious drawback: the observations $q_{i j}$ that are used in this least-squares regression are correlated, and this can lead to the very undesirable situation where the addition of new observations can deteriorate the quality of the estimated parameters. The following example illustrates this point. Consider five equispaced measure points on a straight line: $z_{1}, \ldots, z_{5}$ (see Fig. 3 ) and assume that the true variogram of the field is linear: $\gamma(d ; \alpha)=\alpha d$.

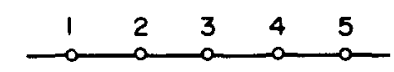

FIG. 3. Five equispaced data points

The least-squares estimate $\hat{\alpha}_{\mathrm{LS}}$ is given by (33)

$$
\hat{\alpha}_{\mathrm{LS}}=\frac{\Sigma \sum q_{i j} d_{i j}}{\Sigma \Sigma \hat{d}_{i j}^{2}} .
$$

As Table 1 demonstrates, adding a third increment to $\left\{q_{13}, q_{35}\right\}$ can either increase or decrease the variance of the estimation error. Note that in this case all overlapping increments are correlated. This very simple example illustrates the problem that arises when one uses a least-squares method with correlated observations. In a 2-D context this problem is even worsened, because with any reasonable variogram model two increments will almost always be correlated. This drawback can be partially removed by the generalized least-squares method that we present now.

\subsection{Estimation of $\alpha$ and $\beta$ by a generalized least-squares method}

Assume as before a variogram of the form $\gamma\left(d_{i j}\right)=\alpha \gamma^{*}\left(d_{i j} ; \beta\right)$. We can now rewrite $(31)$, for $i=1, \ldots, N ; j=i+1, \ldots, N$, in vector notation

$$
\mathbf{Q}=\alpha \boldsymbol{\Gamma}^{*}(\beta)+\mathbf{V}
$$

with the obvious definitions for $\mathbf{Q}, \Gamma^{*}$ and $\mathbf{V}$. Note that $E\{\mathbf{V}\}=0$ by the constant mean assumption on the random field. We call $\mathbf{C}$ the covariance of $\mathbf{V}$

$$
\mathbf{C}=E\left\{\mathbf{V} \mathbf{V}^{\mathrm{T}}\right\} .
$$

TABle 1. Variance of $\hat{\alpha}_{L S} / \alpha$

\begin{tabular}{lc}
\hline $\begin{array}{l}\text { Increments used for } \\
\text { computing } \hat{\alpha}_{\mathrm{LS}}\end{array}$ & VAR $\left\{\frac{\hat{\alpha}_{\mathrm{LS}}-\alpha}{\alpha}\right\}$ \\
\hline$q_{13}, q_{35}$ & 1.0 \\
$q_{13}, q_{35}, q_{25}$ & 1.2 \\
$q_{13}, q_{35}, q_{15}$ & 1.5 \\
$q_{13}, q_{35}, q_{24}$ & 0.9 \\
$q_{12}, q_{23}, q_{34}, q_{45}$ & 0.5 \\
\hline
\end{tabular}


Then the linear minimum variance unbiased (or Markov) estimate $\hat{\alpha}_{M}$ of $\alpha$ is obtained by minimizing the following cost function:

$$
J(\alpha, \beta)=\left\{\mathbf{Q}-\alpha \Gamma^{*}(\beta)\right\}^{\mathrm{T}} C^{-1}\left\{\mathbf{Q}-\alpha \Gamma^{*}(\beta)\right\} .
$$

C is of course a function of $\alpha$ and $\beta$.

If the random field is Gaussian

$$
\begin{aligned}
E\left\{v_{i j} v_{k l}\right\} & =2\left(\gamma_{i l}+\gamma_{j k}-\gamma_{i k}-\gamma_{j l}\right)^{2} \\
& =2 \alpha^{2}\left(\gamma_{i l}^{*}+\gamma_{j k}^{*}-\gamma_{i k}^{*}-\gamma_{j l}^{*}\right)^{2}
\end{aligned}
$$

where the $\gamma^{*}$ s are functions of $\beta$ only. Therefore

$$
\mathrm{C}=\alpha^{2} \Sigma(\beta)
$$

where $\Sigma(\beta)$ is a matrix function of $\beta$ only. In such case the estimation of $\alpha$ and $\beta$ can be decoupled, just as for the ordinary least-squares method

$$
\begin{aligned}
\hat{\alpha}_{\mathrm{M}}=\hat{\alpha}_{\mathrm{M}}(\beta)=\{ & \boldsymbol{\Gamma}^{*}(\beta)^{\mathrm{T}} \Sigma^{-1}(\beta) \\
& \left.\boldsymbol{\Gamma}^{*}(\beta)\right\}^{-1} \Gamma^{*}(\beta)^{\mathrm{T}} \Sigma^{-1}(\beta) \mathrm{Q}
\end{aligned}
$$

and $\hat{\beta}_{\mathrm{M}}$ is obtained by minimizing

$$
J^{*}(\beta)=J\left(\hat{\alpha}_{M}(\beta), \beta\right)
$$

\section{Comments}

(a) If $\beta$ is known, the estimation of $\alpha$ is more robust than with the ordinary least squares. Indeed, one can show that:

(i) $\operatorname{Var}\left\{\hat{\alpha}_{M}\right\} \leqslant \operatorname{Var}\left\{\hat{\alpha}_{L S}\right\}$;

(ii) $\operatorname{Var}\left\{\hat{\alpha}_{M}\right\}$ decreases when new increments are added.

This contrasts with the least-squares method, as illustrated in the previous section

The assumption that $\beta$ is known is not as severe as it might appear because, as we shall see below, $\beta$ can be estimated independently by an interpolation error method.

(b) A drawback of this method is that the matrix $\Sigma(\beta)$, which must be inverted, can become very large. Example: if $N=100$, then the number of increments (which is also the size of $\Sigma(\beta)$ ) is 4950 ! This dimensionality problem, coupled with the problem of correlation between the $q_{i j}$, leads one to think that it might be better to estimate $\alpha$ and $\beta$ using only a subset of the available increments. The selection of a suitable subset of increments is the subject of continuing research. See Gevers and Bastin (1980) for a further discussion of this problem and an application to the case of a linear variogram: $\gamma(d)=\alpha d$.

\subsection{Estimation of $\beta$ by an interpolation error method}

In Sections 4.4 and 4.5 we have estimated $\alpha$ and $\beta$ by adjusting the theoretical variogram to the experimental variogram by least-squares methods. Now if the variogram is estimated in order to design an optimal interpolator, it makes sense to use the minimization of the interpolation errors as a criterion for the estimation of the parameters of the variogram model. This is analogous to the idea of using a prediction error identification method for the estimation of the parameters of a dynamical model when this model is to be used for prediction purposes. This idea is developed in the present section.

Recall that, when the variogram has the form $\gamma(d)=\alpha \gamma^{*}(d ; \beta)$, the optimal interpolation is independent of $\alpha$ (see Section 4.3). The scale factor influences only the interpolation error variance. Therefore, by minimizing some measure of the interpolation error, we shall be able to estimate $\beta$ only. The method proceeds as follows.

(1) At each measure point $(i=1, \ldots, N)$ an optimal estimate $\hat{z}_{i}$ is computed based on the $N-1$ other measure points, using the interpolation formulas of Section 2. In matrix notations, one can write

$$
\hat{\mathbf{Z}}=\boldsymbol{\Lambda}(\beta) \mathbf{Z}
$$

$\Lambda(\beta)$ is a $N \times N$ matrix with zeroes on the diagonal; it depends only on $\beta$ and on the location of the measure points.

(2) A vector of interpolation errors is defined

$$
\mathbf{E} \triangleq \mathbf{Z}-\mathbf{Z}=\left[\mathbf{I}_{N}-\boldsymbol{\Lambda}(\beta)\right] \mathbf{Z}
$$

The mean square interpolation error can then be defined as

$$
\mathbf{E}_{\mathbf{q}} \triangleq\left(\mathbf{E}^{\mathrm{T}} \mathbf{E}\right)^{1 / 2}=\left\{\mathbf{Z}^{\mathrm{T}}[\mathbf{I}-\Lambda(\beta)]^{\mathrm{T}}[\mathbf{I}-\boldsymbol{\Lambda}(\beta)] \mathbf{Z}\right\}^{1 / 2}
$$

(3) The estimate $\hat{\beta}$ is obtained by minimizing $\mathbf{E}_{\mathrm{q}}$ with respect to $\beta$.

\section{Comments}

(a) As will be illustrated in Sections 6 and 7, the interpolation error method gives better results than the least-squares methods, particularly when few data are available.

(b) The drawback of the method is that it does not take into account the geometry of the measure points. One would expect that the interpolation error at a point that is fairly distant from most other measure points would be larger than at a point that lies in a region where the measurements are dense. Yet the criterion (42) gives the same weight to all interpolation errors. Therefore large interpolation errors at the border of the domain will tend to have an unduly large effect on the choice of $\beta$. The 
maximum likelihood method, which we shall now describe, will tend to eliminate this drawback by incorporating the geometry of the measure points.

\subsection{Estimation of $\alpha$ and $\beta$ by a maximum likelihood method}

We assume that the random field is Gaussian. The basic idea of the method is to search the values of $\alpha$ and $\beta$ that maximize the probability density of the vector of interpolation errors $\mathbf{E}$, see (43). Formally, this density is written

$$
f(\mathbf{E})=\left\{(2 \pi)^{N}\left|\mathbf{R}_{\mathrm{E}}\right|\right\}^{-1 / 2} \exp \left\{-\frac{1}{2} \mathbf{E}^{\mathrm{T}} \mathbf{R}_{\mathrm{E}}^{-1} \mathbf{E}\right\}
$$

But, obviously, (45) makes sense only if the covariance matrix $\mathbf{R}_{\mathrm{E}}$ of $\mathbf{E}$ is invertible; this is evidently not the case for the interpolation error vector $\mathbf{E}$ defined by (43). In this case, indeed, the matrix $\left[\mathbf{I}_{N}-\boldsymbol{\Lambda}(\beta)\right]$ is singular and the interpolation errors in $\mathbf{E}$ are not linearly independent.

The solution is to select a subset of $M$ errors among the $N$ available ones in such a way that the covariance is non singular. The vector $\varepsilon$ of these $M$ errors can be written:

$$
\boldsymbol{\varepsilon}=[\overline{\mathbf{\Lambda}}(\beta)-\mathbf{A}] \mathbf{Z}
$$

with $\bar{\Lambda}(\beta)$ a $M \times N$ submatrix of $\boldsymbol{\Lambda}(\beta)$ in (43) and $\mathbf{A}$ is the corresponding $M \times N$ submatrix of $\mathbf{I}_{N}$ in (43).

It is easy to see that the covariance matrix of $\varepsilon$ is of the form

$$
\mathbf{R}_{\varepsilon}=E\left[\boldsymbol{\varepsilon} \varepsilon^{\mathrm{T}}\right]=\alpha \mathbf{R}(\beta)
$$

and the distribution $f(\varepsilon)$ is

$$
f(\boldsymbol{\varepsilon})=\left\{(2 \pi)^{\mathrm{M}} \alpha^{\mathrm{M}} \mid \mathbf{R}(\beta)\right\}^{-1 / 2} \exp \left\{-\frac{1}{2 \alpha} \boldsymbol{\varepsilon}^{\mathrm{T}} \mathbf{R}^{-1}(\beta) \boldsymbol{\varepsilon}\right\} .
$$

The likelihood function $L(\alpha, \beta)$ is given by

$$
\begin{aligned}
L(\alpha, \beta)= & -\log f(\boldsymbol{\varepsilon}) \\
= & \frac{M}{2} \log 2 \pi+\frac{M}{2} \log \alpha \\
& +\frac{1}{2} \log |\mathbf{R}(\beta)|+\frac{1}{2 \alpha} \varepsilon^{\mathrm{T}} \mathbf{R}^{-1}(\beta) \boldsymbol{\varepsilon} .
\end{aligned}
$$

The minimization of $L(\alpha, \beta)$ with respect to $\alpha$ and $\beta$ yields again a decoupled solution:

$$
\hat{\alpha}_{\mathbf{M L}}(\beta)=\frac{1}{\mathbf{M}} \boldsymbol{\varepsilon}^{\mathrm{T}} \mathbf{R}^{-1}(\beta) \boldsymbol{\varepsilon}
$$

while $\hat{\beta}$ is the minimizing value of

$$
L^{*}(\beta)=L\left(\hat{\alpha}_{\mathrm{ML}}(\beta), \beta\right)=\frac{1}{M} \varepsilon^{\mathrm{T}} \mathbf{R}^{-1}(\beta) \varepsilon^{\mathcal{M}} \sqrt{|\mathbf{R}(\beta)|} .
$$

Remark: In the hypothetical case where the interpolation errors are uncorrelated, $\mathbf{R}(\beta)$ is diagonal:

$$
\mathbf{R}(\beta)=\operatorname{diag}\left\{V_{i}^{*}(\beta)\right\}
$$

with $V_{i}^{*}(\beta)$ defined for each error as $V^{*}(\beta)$ in (28). In such case, no matrix inversion is required in (49). In actual applications however, the interpolation errors are never uncorrelated, but, in order to avoid the computation of $\mathbf{R}^{-1}(\beta)$, we shall often replace the ML estimator (49) by a suboptimal estimator derived under the implicit assumption that $\mathbf{R}(\beta)$ is diagonal. We shall call this estimator $\hat{\alpha}_{\mathrm{AML}}$ (for approximate maximum likelihood)

$$
\hat{\alpha}_{\mathrm{AML}}(\beta)=\frac{1}{M} \sum_{i=1}^{M} \frac{\varepsilon_{i}^{2}}{V_{i}^{*}} .
$$

We shall often refer to $\hat{\alpha}_{\mathrm{AML}}$ in later sections.

\subsection{Selection of a variogram model}

The procedure used in practice for the selection of a variogram model among a set of candidate models will be illustrated in the application of Section 6 .

\section{NUMERICAL SIMULATION}

In this section we illustrate, by a simple simulation study, some of the variogram estimation methods which have been described in Section 4. To keep things simple, we consider a 1-D random field generated by the model

$$
Z_{k+1}=Z_{k}+W_{k}
$$

where $k$ denotes the $k$ th point along a straight line; the points are assumed to be equispaced with an elementary space-step of unit length. $W_{k}$ is a zeromean Gaussian white noise with variance $\sigma^{2}=1$. It is well known that $\mathbf{Z}_{k}$ is a discrete Wiener-Levy process with variogram:

$$
\gamma\left(d_{i j}\right)=\frac{\sigma^{2}}{2}|i-j|=0.5 d_{i j} .
$$

Using expression (52), 200 equispaced data have been generated. Out of these a subset of 33 scattered (i.e. non-equispaced) data have been selected. They are listed in Table 2 and they have been used to construct an experimental variogram (Fig. 4). Assuming a variogram model $\gamma(d ; \alpha, \beta)=\alpha d^{\beta}$, the 


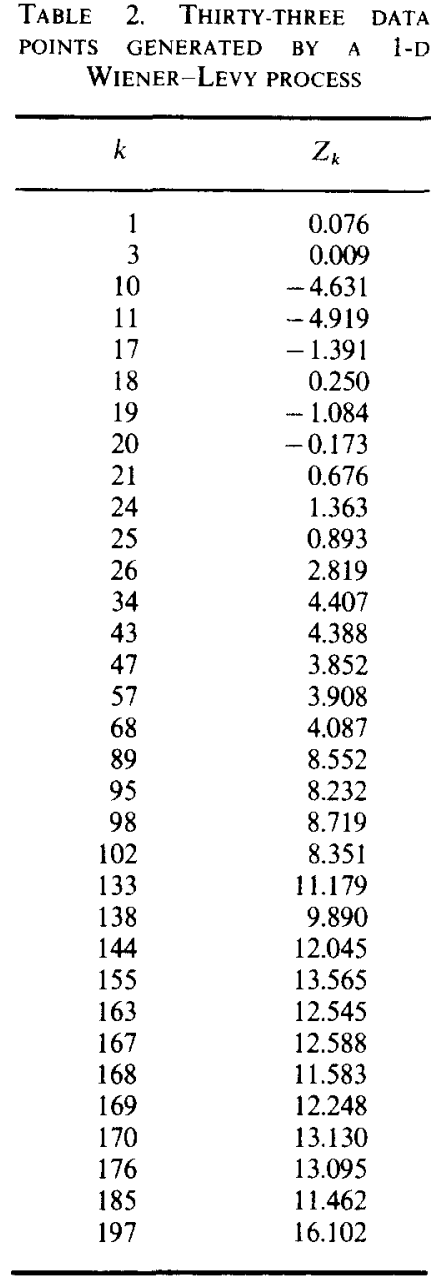

parameters $\alpha$ and $\beta$ have been estimated using the least-squares (LS) and the interpolation (IE) methods described in Section 4. The results are presented in Figs 4 and 5 and in Table 3.

In this table, IE-AML means that $\hat{\beta}$ is computed by the IE method of Section 4.6 and $\hat{\alpha}$ by the approximate maximum likelihood (AML) formula (51). Table 3 also shows the mean square

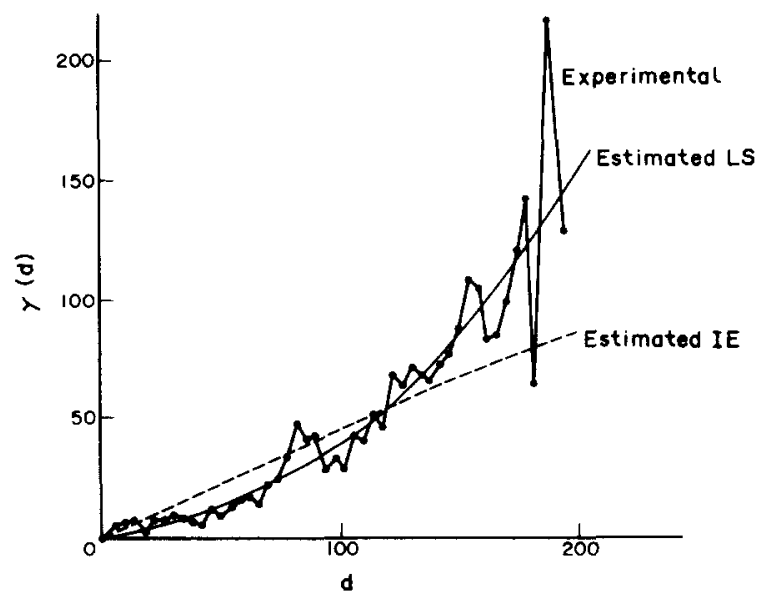

FIG. 4. Experimental and estimated variogram for model (52), (53).

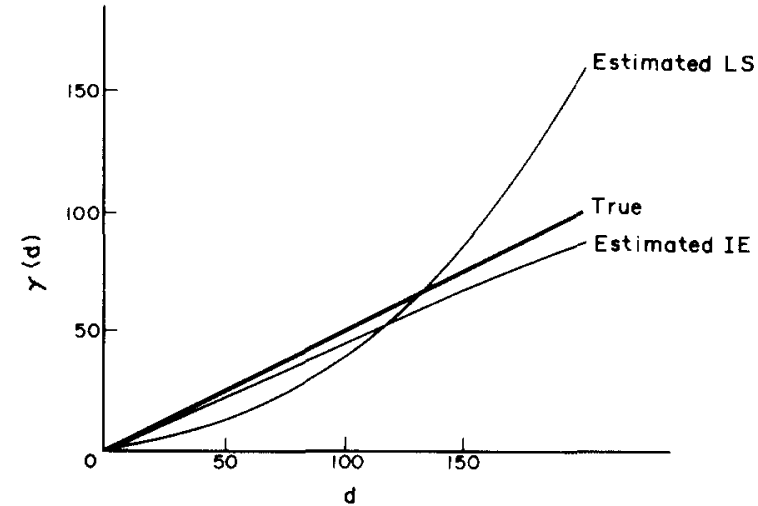

FIG. 5. True and estimated variogram for model (52), (53).

interpolation error $\mathbf{E}_{\mathbf{q}}$, defined by (44), obtained with the true variogram and with each of the estimated variograms.

\section{Discussion}

(1) Notice the erratic behaviour of the experimental variogram.

(2) Clearly the IE method provides a much higher accuracy for the parameter estimates $\hat{\alpha}$ and $\hat{\beta}$ than the LS method (Fig. 5) although the latter gives a better fit to the experimental variogram (Fig. 4).

(3) The mean square interpolation error $\mathbf{E}_{\mathrm{u}}$ obtained with the IE method is lower than that obtained with the LS method. This is to be expected, since with the IE method the interpolation criterion coincides with the variogram model selection criterion.

6. APPLICATION 1: CONTOUR MAPPING OF THE WATER LEVEL IN A GROUNDWATER RESERVOIR

A typical application is the contour mapping of the piezometric level (i.e. the level of the top of the water table) in a groundwater reservoir. Such a contour mapping requires the estimation of the piezometric level at all nodes of the grid covering the domain (in order to establish a chart of the piezometry) from measurements made at a few piezometers scattered within the reservoir (Fig. 6). The piezometric chart is of course of interest per se; sometimes it is also used as input of an identification procedure, whose objective is the modelling of the groundwater flow (Bastin and Duque, 1981) in the aquifer.

TABle 3. Estimation OF THE PARAMETERS $\alpha$ AND $\beta$

\begin{tabular}{llll}
\hline & True & LS & IE-AML \\
\hline$x$ & 0.5 & 0.008 & 0.395 \\
$\beta$ & 1.0 & 1.840 & 0.871 \\
$\mathbf{E}_{\mathrm{q}}$ & 1.3 & 1.45 & 1.29 \\
\hline
\end{tabular}




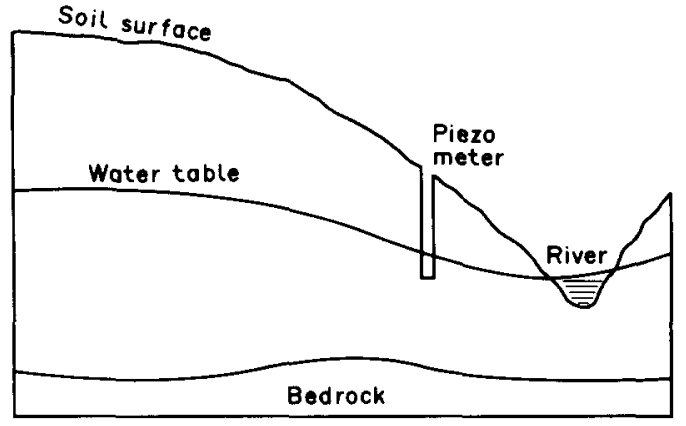

FIG. 6. Groundwater reservoir with piezometer.

Here we shall use this application to illustrate some of the features of the theory presented before: computation of an experimental variogram, choice of a variogram model, estimation of the variogram parameters by different methods, optimal interpolation of the piezometric field in view of contour mapping.

\subsection{Description of the data}

Figures 7 and 8 show the studied domain: a $6 \times 6 \mathrm{~km}$ area around Louvain-la-Neuve (Belgium). Twenty-eight piezometers were available and observed during October and November 1977: they are numbered 1-28 (Fig. 8). The coordinates $\left(x_{i}, y_{i}\right)$ and the measured piezo levels $z\left(x_{i}, y_{i}\right)$ are indicated on Table 4.

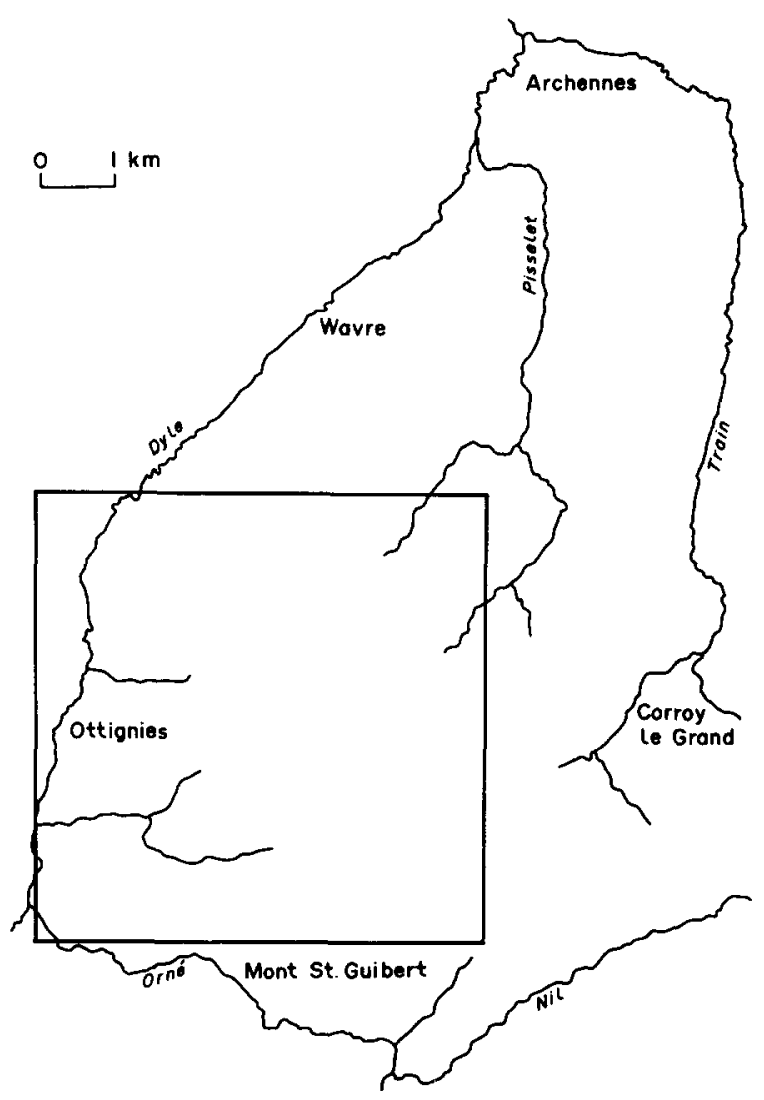

FIG. 7. The Dyle river basin with the $6 \times 6 \mathrm{~km}$ studied domain.

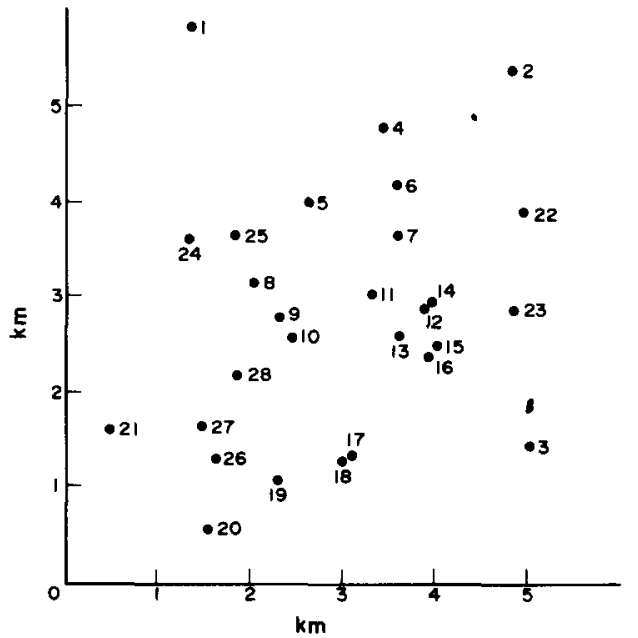

FIG. 8. Location of the 28 piezometers in the studied domain.

\subsection{The experimental variogram}

A distance $d_{\max }=5 \mathrm{~km}$ is considered. This distance has been divided into 50 segments of $100 \mathrm{~m}$ each, and the experimental variogram has been drawn as explained in Section 3. The result has been presented in Fig. 2. This experimental variogram is fairly chaotic, and if it were used as such to compute interpolated values of the piezometry, it would lead to a mean square interpolation error at the data points of $\mathbf{E}_{\mathrm{q}}=171 \mathrm{~m}$. This is totally unacceptable, since the data points are all between 56 and $117 \mathrm{~m}$.

TABLE 4. $(x, y)$ COORDINATES AND MEASURED WATER LEVELS FOR THE 28 PIEZOMETERS OF FIG. 8

\begin{tabular}{|c|c|c|c|}
\hline No. & $\underset{(\mathrm{m})}{X}$ & $\begin{array}{c}Y \\
(\mathrm{~m})\end{array}$ & $\begin{array}{c}Z \\
(\mathrm{~m})\end{array}$ \\
\hline 1 & 165390.0 & 153810.0 & 56.00 \\
\hline 2 & 168860.0 & 153365.0 & 89.47 \\
\hline 3 & 169040.0 & 149456.0 & 116.60 \\
\hline 4 & 167470.0 & 152785.0 & 84.73 \\
\hline 5 & 166650.0 & 152000.0 & 83.00 \\
\hline 6 & 167610.0 & 152175.0 & 89.08 \\
\hline 7 & 167608.0 & 151651.0 & 90.00 \\
\hline 8 & 166057.0 & 151156.0 & 83.12 \\
\hline 9 & 166329.0 & 150786.0 & 86.93 \\
\hline 10 & 166477.0 & 150590.0 & 88.48 \\
\hline 11 & 167345.0 & 151040.0 & 91.20 \\
\hline 12 & 167899.0 & 150891.0 & 107.04 \\
\hline 13 & 167629.0 & 150603.0 & 105.80 \\
\hline 14 & 167972.0 & 150942.0 & 109.17 \\
\hline 15 & 168042.0 & 150499.0 & 110.71 \\
\hline 16 & 167965.0 & 150384.0 & 110.00 \\
\hline 17 & 167115.0 & 149340.0 & 103.66 \\
\hline 18 & 167013.0 & 149312.0 & 104.03 \\
\hline 19 & 166325.0 & 149100.0 & 98.07 \\
\hline 20 & 165575.0 & 148600.0 & 85.50 \\
\hline 21 & 164500.0 & 149625.0 & 60.32 \\
\hline 22 & 168971.0 & 151892.0 & 97.28 \\
\hline 23 & 168871.0 & 150874.0 & 113.80 \\
\hline 24 & 165350.0 & 151625.0 & 60.00 \\
\hline 25 & 165850.0 & 151650.0 & 70.00 \\
\hline 26 & 165650.0 & 149325.0 & 80.00 \\
\hline 27 & 165500.0 & 149675.0 & 70.00 \\
\hline 28 & 165875.0 & 150200.0 & 80.00 \\
\hline
\end{tabular}


This example shows clearly that the use of analytic variograms is absolutely essential. We shall see that with such models the mean-square interpolation error will be of the order of $3 \mathrm{~m}$.

\subsection{Estimation of the parameters $\alpha$ and $\beta$}

Table 5 shows the result of the estimation of the parameters $\alpha$ and $\beta$ for the models (25) and for some combinations of the estimation methods proposed in Section 4.

In Table 5, LS means that $\hat{\alpha}$ and $\hat{\beta}$ are computed by the least-squares method of Section 4.4, IE-AML means that $\hat{\beta}$ is computed by the interpolation Method of Section 4.6 and $\hat{\alpha}$ by the approximate maximum likelihood formula (51), IE-ML means that $\hat{\beta}$ is computed by the IE method and $\hat{\alpha}$ by the maximum likelihood formula (49). Figure 9 illustrates graphically some of the identified models.

\section{Comments}

(1) As can be expected from Fig. 2, only variograms with positive curvature (i.e. $\alpha d^{\beta}$ and $\alpha[1$ $\left.\exp \left(-\beta d^{2}\right)\right]$ ) can be reasonably fitted to the experimental variogram. Indeed both LS and IE methods converge to an estimate $\hat{\beta}=0$ for the models with negative curvature.

(2) With the mean-square interpolation error $\mathbf{E}_{\mathrm{q}}$ as criterion, Table 5 shows that $\alpha d^{\beta}$ is by far superior to $\alpha\left[1-\exp \left(-\beta d^{2}\right)\right]$, even though the latter gives in fact a better least-squares fit to the experimental variogram.

(3) For the model $\alpha d^{\beta}$, we have $\hat{\alpha}_{\mathrm{ML}}=40.2$ and $\hat{\alpha}_{\mathrm{AML}}=31.2 . \hat{\alpha}_{\mathrm{AML}}$, which can be considered as an approximation of $\hat{\alpha}_{\mathrm{ML}}$, is of interest because it is much easier to compute (no matrix inversion). On the other hand, under the assumptions of Section 4.7 , it can be shown that $\left(\hat{\alpha}_{M L}\right)^{-1}$ has a $\chi^{2}$ distribution with $N$ degrees of freedom. This provides a statistical test to compare $\hat{\alpha}_{\mathrm{ML}}$ and $\hat{\alpha}_{\mathrm{AML}}: \hat{\alpha}_{\mathrm{AML}}$ is accepted as an approximation of $\hat{\alpha}_{\mathrm{ML}}$, with $90 \%$ confidence, if the following inequality holds

$$
0.778 \leqslant\left(\frac{\hat{\alpha}_{\mathrm{AML}}}{\hat{\alpha}_{\mathrm{ML}}}\right)^{-1 / 2} \leqslant 1.215 .
$$

It is the case here.

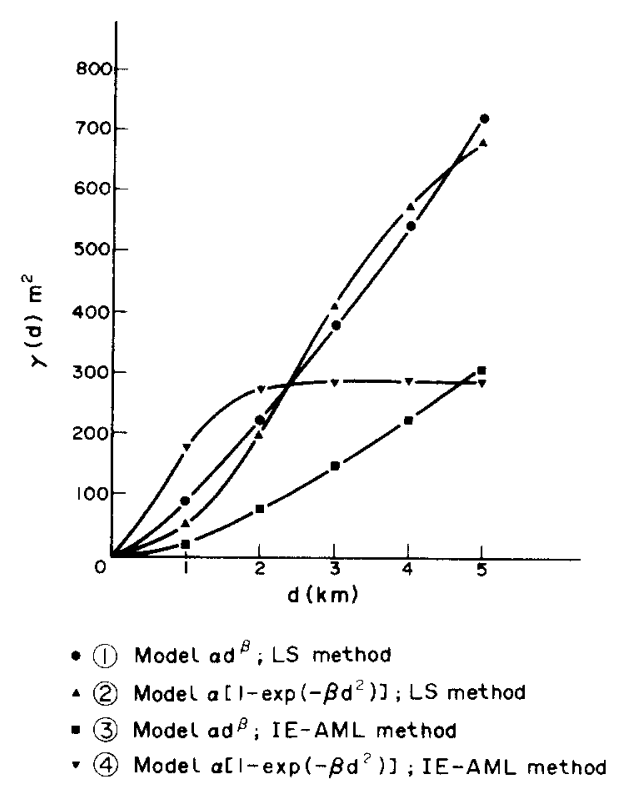

FIG. 9. Estimated variogram models for the piezometric RF.

(4) For the model $\alpha d^{\beta}$, the mean square errors $\mathbf{E}_{\mathrm{q}}$ obtained with the LS and the IE methods are quite close ( 3.27 and 3.01). This does not mean that both models are equivalent: although the interpolated values will be very close, the estimation error variance $s_{0}^{2}$ (which is proportional to $\hat{\alpha}$ : see (27)) will be twice as large with the LS model than with the IE model.

\subsection{Contour mapping of the piezometric field}

To conclude the analysis of the previous section, the model $\gamma(d ; \alpha, \beta)=\alpha d^{\beta}$ is selected with $\alpha=31.2$ and $\beta=1.44$. This model can be used to estimate the piezometric level at any point of $\Omega$ (Fig. 7). A grid with square elements of size $\Delta x=\Delta y=0.5 \mathrm{~km}$ is superimposed on $\Omega$, and $z(x, y)$ is estimated at each node of the grid using the interpolation formulas (13)-(15). A contour map can then be obtained from this grid and is represented in Fig. 10. Since the optimal interpolation technique provides not only piezometric estimates, but also estimation standard deviations, a contour map can also be drawn showing the areas of equal deviations. This is shown

Table 5. Estimation of $\alpha$ and $\beta$ For various vatiogram models

\begin{tabular}{|c|c|c|c|c|c|}
\hline $\begin{array}{l}\text { Variogram } \\
\text { model }\end{array}$ & $\begin{array}{l}\text { Estimation } \\
\text { method }\end{array}$ & $\hat{\alpha}$ & $\beta$ & $\mathbf{E}_{\mathrm{q}}$ & $J_{\mathrm{LS}}$ \\
\hline \multirow[t]{3}{*}{$\alpha d^{B}$} & LS & 91.49 & 1.29 & 3.27 & $3.5110^{7}$ \\
\hline & IE-AML (3) & 31.20 & 1.44 & 3.01 & \\
\hline & IE-ML & 40.18 & 1.44 & 3.01 & \\
\hline \multirow[t]{2}{*}{$\alpha\left[1-\exp \left(\beta d^{2}\right)\right]$} & LS (2) & 792.0 & 0.081 & 13.82 & $3.4910^{7}$ \\
\hline & IE-AML (4) & 277.0 & 0.986 & 5.34 & \\
\hline$\alpha[1-\exp (-\beta d)]$ & LS and IE & & $\beta \rightarrow 0$ & & \\
\hline$\alpha \log (1+\beta d)$ & LS and IE & & $\beta \rightarrow 0$ & & \\
\hline
\end{tabular}

The numbers (1), (2), (3) and (4) refer to Fig. 9. $d$ in $\mathrm{km} \mathbf{E}_{\mathbf{q}}$ in $\mathbf{m}$. 


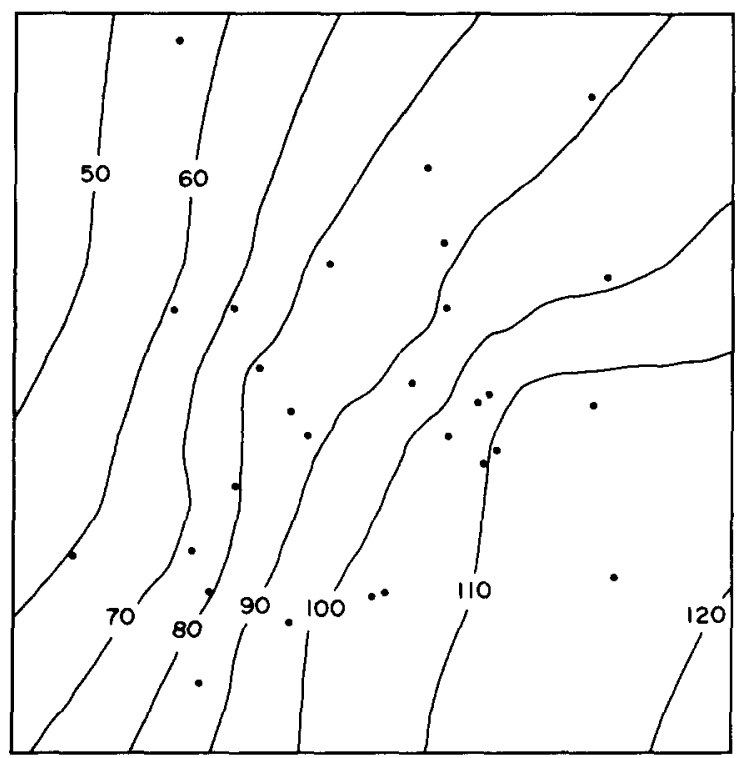

FIG. 10. Contour map of the piezometry for the studied area in Fig. 7, with piezometric levels in meters above sea level. The dots indicate the locations of the piezometers.

in Fig. 11. As could be expected, the standard deviation increases in areas where there are few measure points. In practice, the computation of the experimental variogram, the fitting of various standard variogram models, the optimal interpolation, the computation of $\mathbf{E}_{\mathrm{q}}$ and the contour mapping can be done automatically by a software package KRIGEA/CARTO developed at Louvain University by the Automatic Control Group.

\section{APPLICATION 2: ESTIMATION OF THE AVERAGE RAINFALL OVER A RIVER BASIN}

We consider the problem of estimating the daily average rainfall over a river basin, from daily rainfall

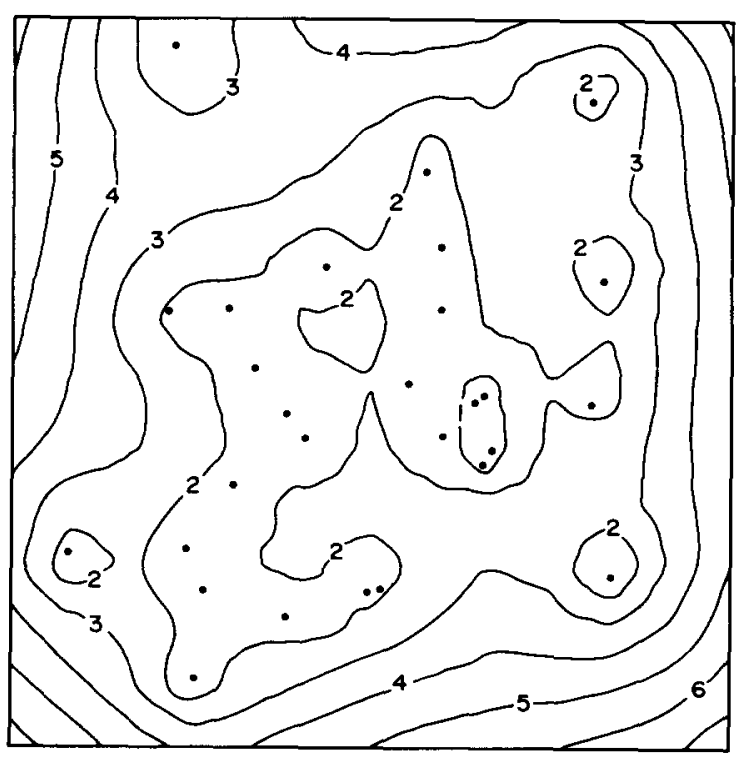

FIG. 11. Contour maps of equal standard deviations for the estimated piezometric levels of Fig. 10. observations made at a few rain-gauges scattered in the basin. This estimate is needed for the development of real-time rainfall-riverflow forecasting models (Lorent and Gevers, 1976).

The 'rainfall function' is denoted $z(k, x, y)$ : it is the volume, per unit area, of precipitated water at the point $(x, y)$ during the day of index $k$. For a fixed $k$, the function $z(k, x, y)$ is viewed as a realization of a 2$\mathrm{D}$ random field denoted $Z(k, x, y)$ whose mean, variance and variogram are assumed spacestationary and denoted:

$$
\begin{gathered}
m(k)=[Z(k, x, y)] \\
\sigma^{2}(k)=E\left\{[Z(k, x, y)-m(k)]^{2}\right\} \\
\gamma(k, i, j)=\frac{1}{2} E\left\{\left[Z\left(k, x_{i}, y_{i}\right)-Z\left(k, x_{j}, y_{j}\right)\right]^{2}\right\} .
\end{gathered}
$$

\subsection{Description of the data}

A map of the Semois river basin $\left(1230 \mathrm{~km}^{2}\right.$, Belgium) is shown in Fig. 12. Seven years (1969-1975) of daily rainfall observations are available in 17 rain-gauges, the locations of which are indicated on the map.

\subsection{Optimal estimation of the average rainfall}

Assume that $N$ rain-gauges, numbered $1-N$, are available in the basin, and denote the observations on day $k$ by

$$
z(k, 1), z(k, 2), \ldots z(k, N) .
$$

Consider a discretization square grid of $M$ nodes, numbered $N+1$ to $N+M$, superimposed on the basin, and denote the (unknown) rainfall at these nodes on day $k$ by

$$
Z(k, N+1), \ldots, Z(k, N+M)
$$

The daily average rainfall over the area (i.e. the basin) is defined as

$$
A(k)=\frac{1}{M} \sum_{j=1}^{M} Z(k, N+j) .
$$

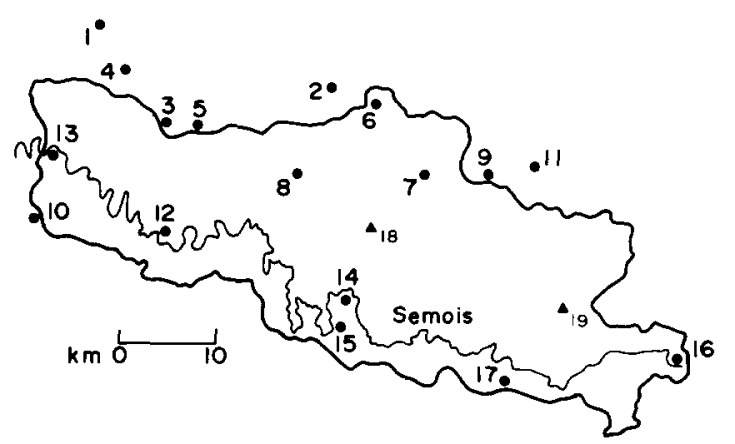

FIG. 12. Semois river basin with locations of the 17 rain-gauges, plus two additional locations $(\boldsymbol{A})$ considered in Section 7.5. 
Note that $A(k)$ is a space-average, not a timeaverage: it is a discrete time series with variance

$$
\sigma_{A}^{2}(k)=\sigma^{2}(k)-\delta_{\mathrm{A}}(k)
$$

where

$$
\delta_{\mathrm{A}}(k)=\frac{1}{M^{2}} \sum_{i=1}^{M} \sum_{j=1}^{M} \gamma(k, N+i, N+j) .
$$

Clearly $\sigma_{\mathrm{A}}^{2}(k)$ is smaller than $\sigma^{2}(k)$, since $\delta_{\mathrm{A}}(k)$ is positive. By a straightforward extension of Section 2, a linear minimum variance estimator of $A(k)$ can be obtained from the measurements $z(k, 1), \ldots, z(k, N)$

$$
\hat{A}(k)=\sum_{i=1}^{M} \lambda_{i} z(k, i)
$$

The coefficients $\lambda_{i}$ are the solution of the linear system

$$
\begin{aligned}
& \sum_{j=1}^{N} \lambda_{j} \gamma(k, i, j)+\mu=\frac{1}{M} \sum_{j=1}^{M} \gamma(k, i, N+j) \\
& \quad i=1, \ldots, N \\
& \sum_{j=1}^{M} \lambda_{j}=1 .
\end{aligned}
$$

The estimation error variance is written

$$
\begin{aligned}
\sigma_{\mathrm{E}}^{2}(k) & =E\left\{[A(k)-\hat{A}(k)]^{2}\right\} \\
& =\mu-\delta_{\mathrm{A}}(k)+\frac{1}{M} \sum_{i=1}^{M} \sum_{j=1}^{M} \lambda_{i} \gamma(k, i, N+j) .
\end{aligned}
$$

\subsection{Identification of monthly variogram models}

In order to take the potential seasonal trends of the rainfall into account, we assume a piecewise stationary seasonal trend (on a monthly basis) for the rainfall RF. More precisely we assume that the variogram $\gamma(k, i, j)$ is time invariant during one month, but not necessarily from one month to another. Furthermore we assume that the time nonstationarity of the RF is periodic with a period of one year. Then, all the available rainfall events are divided into 12 classes, one class for each month: for example, the data of November 1969, November $1970, \ldots$, November 1975 are taken in the same class and processed together for the estimation of a variogram model.

Notice that, in this particular application, several hundred realizations of the $R F$ are available in each class, since there are several thousand available rainfall events: this enables us to have more confidence in the experimental variograms and to expect more robustness from the LS variogram estimation method. A detailed discussion of the variogram identification can be found in Lorent $e t$ al. (1982). In this brief report we give only the main result. The selected variogram is of the form

$$
\gamma\left(l, d_{i j}\right)=\alpha(l) d_{i j}^{\beta} \quad l=1, \ldots, 12
$$

The estimate of $\beta$, obtained by LS, is $\hat{\beta}=0.51$. The monthly values of $\hat{\alpha}(l)$ are shown in Table 6 and illustrated in Fig. 13. These results clearly show the seasonal behaviour of the rainfall field: in summer, local storm showers tend to produce large spatial variations in rainfall, while in winter the rain is more homogeneously distributed over the whole catchment area.

\subsection{Identification of daily variogram models}

Our analysis has shown that choosing a unique model within a given month would lead to a systematic bias of the estimation variance: a systematic underevaluation of $\sigma_{\mathrm{E}}^{2}$ for high-intensity rainfalls and an overevaluation for low-intensity rainfalls (see Lorent et al., 1982). Therefore, we have been led to a variogram of the form

$$
\gamma\left(k, d_{i j}\right)=\alpha(k) d_{i j}^{\beta}
$$

with $\hat{\beta}=0.51$ as in (64), but where $\hat{\alpha}(k)$ is estimated daily by a least-squares fit to the cluster of points corresponding to the experimental variogram of that particular day. The structure (65) of the variogram has important implications for the computation of $\hat{A}(k)$ and $\sigma_{\mathrm{E}}^{2}(k)$.

(1) It can be shown that the coefficients $\lambda_{i}$ of $\hat{A}(k)$

\begin{tabular}{|c|c|}
\hline$k$ & $x(k)$ \\
\hline January & 0.23 \\
\hline February & 0.31 \\
\hline March & 0.34 \\
\hline April & 0.30 \\
\hline May & 0.69 \\
\hline June & 1.05 \\
\hline July & 1.59 \\
\hline August & 1.11 \\
\hline September & 0.57 \\
\hline October & 0.48 \\
\hline November & 0.19 \\
\hline December & 0.35 \\
\hline
\end{tabular}
(see (61)) are independent of $\alpha(k)$ and consequently time-invariant. Hence $\hat{A}(k)$ turns out to be a unique time-invariant weighted sum of the observations $z(k, i)$, where the weighting coefficients depend only upon the geometrical location of the rain-gauges and can be computed once and for all.

(2) It can also be shown that

$$
\sigma_{\mathrm{E}}^{2}(\mathrm{k})=\alpha(k) V_{\mathrm{E}}^{*}
$$




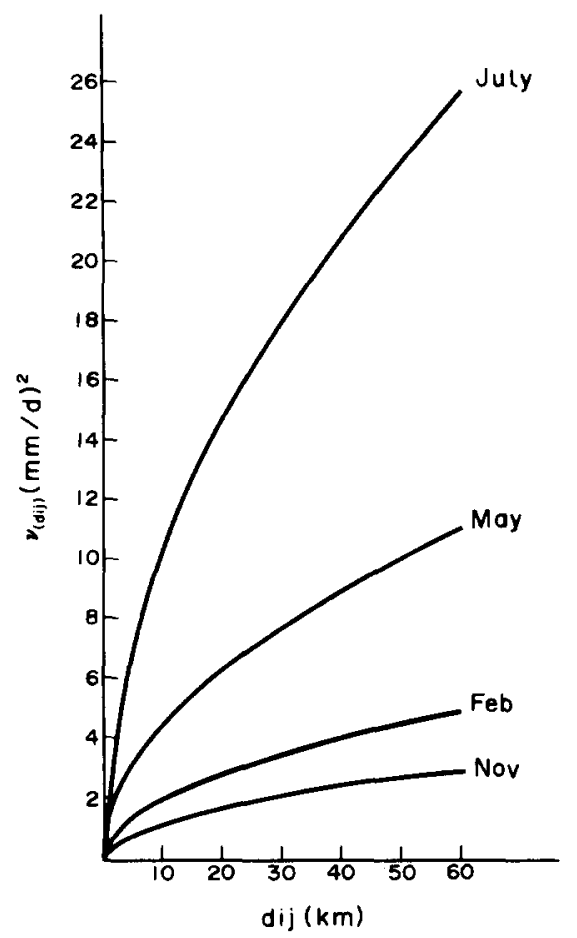

FiG. 13. Estimated monthly variograms.

with $V_{E}^{*}$ a normalized time-invariant variance. The time dependence of $\sigma_{\mathrm{E}}^{2}(k)$ is only through $\alpha(k)$.

Therefore, the practical procedure for the daily computation of the average areal rainfall is as follows:

(a) compute, once and for all, the weighting factors $\lambda_{i}$ and the normalized error variance $V_{E}^{*}$;

(b) for each day $k$

(i) compute $\hat{A}(k)=\sum_{i=1}^{N} \lambda_{i} z(k, i)$;
TAble 7. Semois River basin. WEIGHTING COEFFICIENTS OF THE OPTIMAL RAINFALL ESTIMATOR

\begin{tabular}{cc}
\hline $\begin{array}{c}\text { Rain-gauge } \\
\text { No. }\end{array}$ & $\begin{array}{c}\lambda_{i} \\
(\%)\end{array}$ \\
\hline 1 & 0.8 \\
2 & 2.0 \\
3 & 3.8 \\
4 & 2.0 \\
5 & 3.6 \\
6 & 3.3 \\
7 & 8.5 \\
8 & 9.6 \\
9 & 6.5 \\
10 & 3.9 \\
11 & 5.2 \\
12 & 8.9 \\
13 & 4.0 \\
14 & 9.8 \\
15 & 7.4 \\
16 & 8.6 \\
17 & 12.1 \\
$V$ & 0.48 \\
\hline
\end{tabular}

(ii) compute $\alpha(k)$ by least-squares fit to the experimental variogram; and

(iii) compute $\hat{\sigma}_{\mathrm{E}}^{2}(k)=\hat{\alpha}(k) V_{\mathrm{E}}^{*}$.

With this procedure, the time-nonstationarity of the rainfall field is concentrated in the time-varying parameter $\alpha(k)$ which takes into account both the seasonal and the intensity variations. Tables 7 and 8 show some typical results of this estimation procedure.

\subsection{Optimal selection of rain-gauge locations}

Once the variogram parameter model has been fixed, the normalized variance $V_{\mathrm{E}}^{*}$ can be viewed as

TABle 8. Semois RIVER basin. ESTIMATION RESUltS FOR SOME DAYS CHOSEN IN THE YEAR 1971

\begin{tabular}{cccccc}
\hline $\begin{array}{c}\text { Rain-gauge } \\
\text { No. }\end{array}$ & $26 / 1$ & $26 / 4$ & $18 / 6$ & $16 / 8$ & $19 / 12$ \\
\hline 1 & 33.7 & 14.4 & 27.0 & 14.2 & 11.4 \\
2 & 34.7 & 17.2 & 26.0 & 11.4 & 11.8 \\
3 & 32.3 & 16.3 & 31.6 & 24.0 & 12.9 \\
4 & 29.0 & 15.5 & 34.2 & 14.2 & 16.3 \\
5 & 33.8 & 16.6 & 33.6 & 20.0 & 13.2 \\
6 & 32.0 & 16.8 & 35.2 & 9.1 & 9.8 \\
7 & 30.3 & 20.0 & 26.4 & 7.7 & 9.5 \\
8 & 35.8 & 17.8 & 32.3 & 12.3 & 11.2 \\
9 & 31.5 & 15.7 & 19.3 & 8.2 & 10.0 \\
10 & 28.4 & 15.6 & 41.8 & 23.2 & 7.3 \\
11 & 33.9 & 20.7 & 28.2 & 7.0 & 12.4 \\
12 & 39.5 & 19.6 & 39.6 & 22.8 & 9.2 \\
13 & 35.3 & 12.8 & 29.3 & 24.5 & 12.8 \\
14 & 28.2 & 24.5 & 32.9 & 11.0 & 12.0 \\
15 & 24.5 & 23.1 & 38.4 & 11.1 & 8.0 \\
16 & 31.0 & 21.4 & 24.3 & 7.8 & 11.1 \\
17 & 30.9 & 21.5 & 29.9 & 8.0 & 13.3 \\
Estimation results & & & & & \\
$\hat{A}$ & 31.9 & 19.5 & 30.9 & 12.7 & 11.1 \\
$\hat{\alpha}$ & 2.09 & 2.08 & 6.39 & 8.30 & 0.85 \\
$\sigma_{\mathrm{E}}$ & 0.99 & 0.98 & 1.73 & 1.97 & 0.63 \\
$\sigma_{\mathrm{E}} / \hat{A}$ & $3.1 \%$ & $5.0 \%$ & $5.6 \%$ & $15.5 \%$ & $5.7 \%$ \\
\hline
\end{tabular}

Data are $\mathrm{mm} / \mathrm{day}$. 
depending exclusively on the location of the raingauge locations. Therefore, $V_{\mathrm{E}}^{*}$ is an efficient tool for solving rain-gauge allocation problems. We illustrate this point by showing an iterative selection of the most representative rain-gauges in the basin. Two potential supplementary rain-gauges (numbered 18 and 19) are added to the seven existing ones (Fig. 12). For each of the 19 locations, we can compute $V_{E}^{*}$ as if each of these locations was the only one available, and select the one that leads to the smallest $V_{E}^{*}$. Next we can add to this first gauge a second station which, combined with the first one, leads to a minimum $V_{\mathrm{E}}^{*}$ again. This procedure can be continued, adding more stations and monitoring the decrease of $V_{\mathrm{E}}^{*}$, until the obtained precision is judged satisfactory. The result is shown in Fig. 14. We notice that:

(a) The last seven rain-gauges chosen (Nos. $6,10,5,11,4,2,1)$ are obviously superfluous, since including them in the optimal estimator does not result in any significative decrease of $V_{\mathrm{E}}^{*}$.

(b) The two supplementary locations (18 and 19), where no measurements had been collected, are clearly among the best possible locations. Following our study of the rainfall in the Semois river basin, two additional rain-gauges were installed in these two locations.

\section{CONCLUSIONS}

We have given a self-contained and hopefully informative presentation of the interpolation problem in 2-D random fields with scattered data. We have shown that the major difficulty in applying Best Linear Unbiased Estimators to real life 2-D problems is the estimation of a model for the RF. To obtain meaningful results in applications where the data are scarce, this model must take the form of an analytic expression for the spatial covariance or for the spatial variogram. We have argued that the variogram is preferable, mainly because it covers

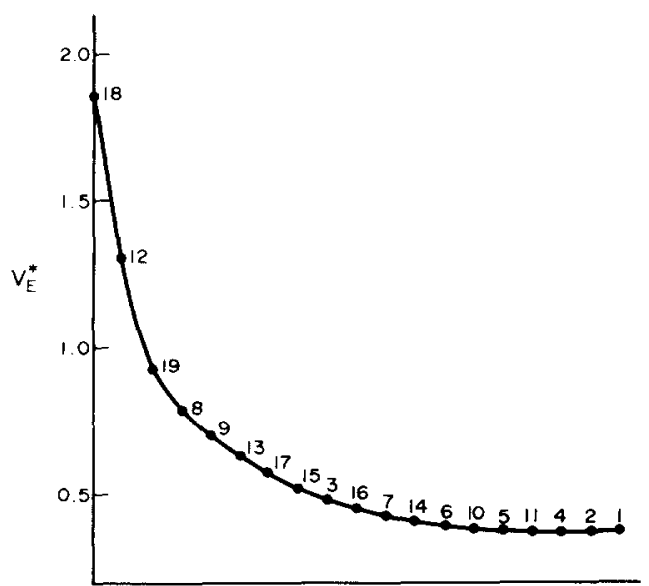

FIG. 14. Iterative selection of the rain-gauge locations. random fields with stationary increments, but also because it is as efficient as the covariance in practical applications to presumed stationary fields. The problem then becomes one of choosing an appropriate variogram model and of estimating its parameters. We have defined the class of admissible variogram models, and examined several parameter estimation techniques. We have illustrated these techniques with two applications, both drawn from the field of hydrology but very different technically. From the experimental results we have concluded that the IE and $M L$ methods seem to be more robust than the LS method for the estimation of $\beta$ and that the mean square error $\mathbf{E}_{\mathbf{q}}$ turns out to be better than the LS criterion $J$ for the selection of a variogram model among a set of candidate models.

Certainly there is room for refinements and further theoretical developments. We may mention the following points which are the matter of our present research:

--theoretical analysis of the robustness of the identification methods with small data sets;

convergence analysis of the identification methods for large data sets (the difficulty here is to give a precise meaning to the notion of a 'large' data set in a 2-D context):

- use of cross validation to validate the variogram models (in our applications, cross validation was almost impossible due to the scarcity of the data points: 28 in the piezometric application, 16 in the rainfall application; examples of cross validation can be found in Lebel and Bastin, in press;

-- tests of stationarity in RF with scarce and nonequispaced data points.

We hope the reader is by now convinced that this 2$D$ interpolation technique has a wide range of potential applications.

Acknowledgements---The authors wish to thank the Fondation Universitaire Luxembourgeoise and the Fonds de Developpement Scientifique (UCL) for providing the support for this research, B. Lorent and the members of the Department de Genie Rural (UCL) for providing the data and for useful discussions, and also $\mathrm{C}$. Obled for some interesting discussions.

\section{REFERENCES}

Anderson, B. D. O. and J. B. Moore (1979). Optimal Filtering Prentice-Hall, New York.

Bastin, G. (1979a). Stochastic parameter estimation of a steadystate groundwater flow model. In Simulation of Systems, Proc IMACS Congress, pp. 471 477. North-Holland. Amsterdam.

Bastin, G. (1979b). Approches deterministe et stochastique pour l'identification de systems hydrogeologiques, Ph.D. thesis. Louvain University.

Bastin, G. (1981). Identification of steady-state distributed systems with space-variable parameters. In Proc. 8 th IF $A C$ World Congress, Kyoto, Japan, vol. 11, pp. 64-69.

Bastin, G. and C. Duque (1981). Modelling of steady-state groundwater flow systems: deterministic and stochastic approaches. In Proc. IASTED Symp. on Modelling. Identification and Control, Davos, pp. 10-16.

Bastin, G. and M. Gevers (1978). Joint use of space interpolation and optimization methods for steady-state aquifer modeling 
with scarce data. In Modeling, Identification and Control in Environmental Systems, pp. 427-441. North-Holland Amsterdam.

Bastin, G. and E. Henriet (1982). A minimum variance property of Levinson predictors using variograms. Syst Control Lett., 2, 25.

Chirlin, G. R. and G. Dagan (1980). Theoretical head variograms for steady flow in statistically homogeneous aquifers. Water Resourc. Res., December 1980.

Chua, S. H. and R. L. Bras (1982). Optimal estimators of mean areal precipitations in regions of orographic influence. $J$. Hydrol. 57, 23.

Cramer, H. and M. R. Leadbetter (1967). Stationary and Related Stochastic Processes. Wiley, New York.

Creutin, J. D. and Ch. Obled (1982). Objective analyses and mapping techniques for rainfall fields: an objective comparison. Water Resourc. Res. 18, 413.

Darricau, H. (1979). Nappe du calcaire carbonifere, traitement geostatistique de données, Ecole des Mines de Paris, Centre d'Informatique Geologique.

Darricau, H. (1980). Recherche d'un modele de Krigeage pour l'estimation of pluies Cevenoles, Ecole des Mines de Paris, Centre d'Informative Geologique.

David, M. (1977). Geostatistical Ore Reserve Estimation. Elsevier, Amsterdam.

Delfiner, P. and J. P. Delhomme (1975). Optimum interpolation by Kriging. In Display and Analysis of Spatial Data. J. C. Davis and M. J. McCullogh (Eds). Wiley, New York.

Delhomme, J. P. (1976). Applications de la theorie des variables regionalisees dans les sciences de l'eau. Doctoral dissertation, Universite de Paris-VI.

Delhomme, J. P. (1978). Kriging in the hydrosciences. Adv. Water Resourc. 1, 251.

Delhomme, J. P. (1979), Spatial variability and uncertainty in groundwater-flow parameters: A geostatistical approach, Water Resourc. Res., 15, 269.

De Wrachien, D. (1976). Hydrological maps: Automatic contouring and reliability. Int. Autom. Instrum. Conf., Milan.

Gambolati, G. and G. Volpi (1979). Groundwater contour mapping in Venice by stochastic interpolators. Water Resourc. Res., 15, 281.

Gevers, M. (1984). On the use of variograms in Levinson predictors. In A. Bensoussan and J. L. Lions (Eds). Analysis and
Optimization of Systems, Part 2, pp. 268-278. Springer Verlag, Berlin.

Gevers, M. and G. Bastin (1980). On the estimation of the variogram in spatial interpolation methods used in groundwaterflow modelling. In D. G. Lainiotis and N. S Tzannes (Eds), Applications of Information and Control Systems, pp. 60-68. Reidel.

Gevers, M. and G. Bastin (1982). What does system identification have to offer? Proc. 6th IF AC Symp. on Identification and System Parameter Estimation, Washington D.C., vol. 1, pp. 29-36.

Journel, A. G. and Ch. J. Huijbrechts (1978). Mining Geostatistics. Academic Press, New York.

Kitanidis, P. K. (1983). Statistical estimation of polynomial generalized covariance functions and hydrologic applications. Water Resourc. Res., 19, 909.

Krige, D. G. (1951). A statistical approach to some mine valuations and allied problems on the Witwatersrand. Master's thesis, Univ. of Witwatersrand.

Lebel, T. and G. Bastin. Variogram identification by the mean squared interpolation error method. $J$. Hydrol., in press.

Lorent, B., G. Bastin, C. Duque and M. Gevers (1982). Optima estimation of the average areal rainfall over a basin and optimal selection of raingauges locations. Proc. 6th IFAC Symp. on Identification and System Parameter Estimation, Washington D.C., vol. 2, pp. 1403-1408.

Lorent, B. and M. Gevers (1976). Identification of a rainfallrunoff process; application to the Semois river (Belgium). Proc 4th IFAC Symp. on Identification and System Parameter Estimation, Tbilisi, USSR.

Matheron, G. (1965). Les variables regionalisees et leur estimation. Masson, Paris.

Matheron, G. (1971). The theory of regionalized variables and its applications. Cahiers du Centre de Morphologie Mathématique, Ecole des mines de Paris, Fontainebleau.

Matheron, G. (1973). The intrinsic random functions and their applications. Adv. Appl. Prob., 5, 439.

Papoulis, A. (1965). Probability, Random Variables and Stochastic Processes. McGraw-Hill, New York.

Volpi, G. and G. Gambolati (1978), On the use of a main trend for the Kriging technique in hydrology. Adv. Water Resourc., 1 , 345 . 